Artículo Original

\title{
Diseño y validación de una escala de estrés financiero en trabajadores dependientes peruanos durante la pandemia del COVID-19 (EFT-Cov19)
}

\section{Design and validation of a financial stress scale in Peruvian dependent workers during the COVID-19 pandemic (EFT-Cov19)}

https://doi.org/10.52808/bmsa.7e5.61e2.021

Renzo Felipe Carranza Esteban ${ }^{1}$

https://orcid.org/0000-0003-4059-8062

Oscar Mamani-Benito ${ }^{2}$

https://orcid.org/0000-0002-9818-2601

J. Franco Rodriguez-Alarcón ${ }^{3,4}$

https://orcid.org/0000-0003-4059-8214

Avelino Sebastian Villafuerte De La Cruz ${ }^{5}$

https://orcid.org/0000-0002-9447-8683

Dennis Arias-Chávez 6

https://orcid.org/0000-0003-1500-8366

Christian R. Mejia ${ }^{7 *}$

https://orcid.org/0000-0002-5940-7281

Recibido: 11/05/2021

Aceptado: 23/07/2021

\section{RESUMEN}

La pandemia del COVID-19 ha generado estragos en la vida de los trabajadores de diferentes partes del mundo. La inestabilidad propia de esta etapa de emergencia sanitaria ha repercutido en la salud mental de esta población. El objetivo fue evaluar las propiedades psicométricas de una escala de estrés financiero para trabajadores dependientes peruanos. Estudio observacional, analítico, instrumental y transversal en 749 trabajadores. Se realizó un Análisis Factorial Exploratorio (AFE), por mínimos cuadrados no ponderados, previo análisis de la prueba de Bartlett y el coeficiente KaiserMeyer-Olkin (KMO). La bondad de ajuste absoluta e incremental se determinó a través del índice de ajuste comparativo (CFI) y el índice de TuckerLewis Index (TLI). Se realizó un AFE previo análisis del índice Kaiser-Meyer-Olkin (KMO = 0,903) y la prueba de esfericidad de Bartlett (1751.9; gl $=36 ; \mathrm{p}<0,001)$, los cuales fueron adecuados. Los ítems convergieron en un solo factor. La EFT-Cov19 correlacionó positivamente con la LABORPE $(r=0,564, p<0,01)$ y con un tamaño del efecto mediano. La fiabilidad de la EFT-Cov19 se calculó con el coeficiente $\alpha$ de Cronbach, obteniendo un valor aceptable $(\alpha=0,896$; IC 95\% =0,88-0,90). En conclusión, la escala EFT-Cov19 es una escala valida, confiable y adecuada para medir el estrés financiero en trabajadores dependientes durante la pandemia del COVID-19.

Palabras clave: estudio de validación, salud ocupacional, COVID-19, Perú.

\begin{abstract}
The COVID-19 pandemic has wreaked havoc in the lives of workers in different parts of the world. The instability inherent to this stage of health emergency has had repercussions on the mental health of this population. The aim was to evaluate the psychometric properties of a financial stress scale for Peruvian dependent workers. Observational, analytical, instrumental and cross-sectional study in 749 workers. An exploratory factor analysis (EFA), by unweighted least squares, was performed after analysis of Bartlett's test and the Kaiser-Meyer-Olkin coefficient (KMO). The absolute and incremental goodness of fit was determined by means of the comparative fit index (CFI) and the Tucker-Lewis Index (TLI). A PFA was performed after analysis of the Kaiser-Meyer-Olkin index $(\mathrm{KMO}=0.903)$ and Bartlett's test of sphericity $(1751.9 ; \mathrm{gl}=36 ; \mathrm{p}\langle 0.001)$, which were adequate. The items converged into a single factor. The EFT-Cov19 correlated positively with the LABOR-PE $(r=0.564, p<0.01)$ and with a medium effect size. The reliability of the EFT-Cov19 was calculated with Cronbach's $\alpha$ coefficient, obtaining an acceptable value ( $\alpha=0.896 ; 95 \%$ CI $=0.88-0.90)$. In conclusion, the EFT-Cov19 scale is a valid, reliable and adequate scale to measure financial stress in dependent workers during the COVID-19 pandemic.
\end{abstract}

Key words: validation study, occupational health, COVID-19, Peru.

1. Facultad de Humanidades, Universidad San Ignacio de Loyola. Lima, Perú 2. Universidad Peruana Unión. Juliaca, Perú. 3. Asociación Médica de Investigación y Servicios en Salud. Lima, Perú. 4. Facultad de Medicina Humana "Manuel Huamán Guerrero". Universidad Ricardo Palma. Lima, Perú. 5. Universidad Peruana Unión. Tarapoto, San Martín, Perú. 6. Universidad Continental. Arequipa, Perú. 7. Centro de Investigación en Medicina Traslacional. Universidad Norbert Wiener. Lima, Perú. 


\section{Introducción}

La población de trabajadores dependientes se ha visto seriamente afectada a causa del impacto de la pandemia del COVID-19 (Organización Internacional del Trabajo, 2020a). Generando repercusiones en su salud mental debido a la inseguridad laboral, la inestabilidad económica, las restricciones sociales y comerciales, entre otras (Mamani-Benito et al., 2020). Según informes de la Organización Internacional del Trabajo (Organización Internacional del Trabajo, 2020a) el estrés ha sido una de las principales manifestaciones psicológicas, a causa de la incertidumbre vivida durante la emergencia sanitaria; dando lugar a un escenario de posibles riesgos respecto al grado de precisión en las funciones laborales e incremento de la posibilidad de cometer errores, aumentando así la probabilidad de que se produzcan lesiones y accidentes en el trabajo (Organización Internacional del Trabajo, 2020b).

En base a lo hallado en la literatura científica existen grupos de trabajadores con mayor riesgo psicosocial, como por ejemplo los profesionales de la salud (Ceballos-Vásquez et al., 2020), que, en un estudio realizado en Colombia, más del $67 \%$ de quienes fueron designados a primera línea experimentaron niveles moderados y altos de Burnout (Vásquez-Trespalacios et al., 2020). Ante ello, la comunidad científica y las instituciones de salud ya vienen implementando medidas preventivas para mejorar sus condiciones laborales (Delgado-Fernández et al., 2021). A diferencia de este grupo, trabajadores de otros sectores como educación, transporte, comercio, infraestructura entre otros, aun dependen de la gestión ocupacional que puedan impulsar las empresas donde trabajan, para mitigar alteraciones psicológicas producidas por la incertidumbre laboral. Esto ocasiona que experimenten sobre todo estrés financiero (Alola et al., 2020; Robillard et al., 2020), tal como lo revela un estudio donde se muestra que la pérdida de ingresos y tensión financiera se asoció con los síntomas depresivos (Hertz-Palmor et al., 2021) y hasta el riesgo de estrés post traumático según la ocupación (Mejia et al., 2020).

Aunque la investigación en el contexto de las empresas y las crisis es limitada, con la aparición de la pandemia COVID-19 se ha impulsado la generación de nuevas evidencias (Doern, 2021); de este modo, la literatura da cuenta de que una de las manifestaciones comunes en trabajadores dependientes en América Latina es el estrés financiero (Robillard et al., 2020), entendido como una sensación subjetiva negativa que percibe una persona al ser incapaz de satisfacer demandas de tipo económico, pagar cuentas y disponer de fondos monetarios para subsistir mensualmente (Mejía Córdova et al., 2017a).

En el ámbito de la salud ocupacional, la evaluación del estrés es una prioridad para prevenir trastornos psicológicos mayores, como la ansiedad generalizada, estrés postraumático y hasta el mismo síndrome de Burnout, factores que podrían perjudicar la productividad laboral (Ganga Contreras \& Villacís Moyano, 2018). Por ello, es indispensable contar con instrumentos de medición documental debidamente válidos y confiables; más aún, en el contexto actual, donde sufrir repercusiones psicológicas a causa de la COVID-19 parece común. Ante ello, una revisión en la literatura científica revela la existencia de algunas alternativas como la escala de Hernández et al. (Hernández González et al., 2012) que mide estrés laboral en profesionales de la salud; así también, la escala de Campo-Arias et al. (Campo-Arias et al., 2020) adaptada para el contexto de la COVID-19 y orientada a población general; sin embargo, aún no se cuenta con un instrumento ideal para evaluar el estrés financiero, variable que está tomando importancia en la comunidad científica (Robillard et al., 2020), debido a su relación con la pérdida de productividad (Mejía Córdova et al., 2017a) y el bienestar psicológico (Mejía Córdova, 2017b). Por lo que el objetivo fue el evaluar las propiedades psicométricas de una escala de estrés financiero para trabajadores dependientes peruanos.

\section{Materiales y Métodos}

El diseño es observacional, analítico, instrumental y de corte transversal (Ato et al., 2013). Se usó el muestreo no probabilístico de tipo bola de nieve, a través del cual se contó con la participación de 749 trabajadores peruanos dependientes (58,2\% mujeres y 41,8\% varones), cuyas edades oscilaron entre los 18 y 63 años $(\mathrm{M}=32,98$; $\mathrm{DE}=10,29$ ) de los cuales el 76,4\% presentó un grado de instrucción superior. Es importante mencionar que estos encuestados provenían en su mayoría de la capital (Lima Metropolitana), de sectores nétamente urbanos y que tenían acceso rápido a internet (debido que mediante esta vía se realizó la encuesta).

En el proceso de investigación se consideró la revisión bibliográfica en las bases de datos SciELO y Scopus, esto para identificar y sintetizar el constructo, elaborar el marco teórico y definir conceptualmente. Se diseñó la escala EFT-Cov19; que está compuesta por 9 ítems, cuyas opciones de respuesta fueron: completamente en desacuerdo, en desacuerdo, ni de acuerdo ni en desacuerdo, de acuerdo y totalmente de acuerdo. Además, se empleó 2 instrumentos para analizar la evidencia de validez basada en otras variables. Por lo que, se consideró la escala de inseguridad laboral percibida (LABOR-PE-COVID-19) diseñada y validada en trabajadores peruanos propuesto por Mamani et al. (2020) con un coeficiente de alfa de Cronbach de 0,765 (IC95\%, 0,73-0,79) (Mamani-Benito et al., 2020). Por otro lado, también con el fin de comparación, se utilizó The World Health Organization - Five Well-Being Index (WHO-5) que es un cuestionario conformado por 5 ítems con alternativas de respuesta tipo Likert $(0=$ nunca, $1=$ a veces, $2=$ muchas veces, 3 = siempre) cuyo objetivo es explorar el bienestar general. Los que obtienen mayor puntaje tiene mayor 
bienestar, mientras que los de menor desempeño tienen evidencia de síntomas depresivos importantes. El coeficiente alfa de Cronbach fue determinado como evidencia de confiabilidad, obteniendo un valor global de 0,889 (IC $95 \%$ : 0,87 - 0,90) (Topp et al., 2015).

Para el análisis de datos, en la primera etapa se analizó los estadísticos descriptivos de los ítems de la escala EFT-Cov19 (media, desviación estándar, asimetría y curtosis). En la segunda etapa se realizó un Análisis Factorial Exploratorio (AFE), esto por la técnica de los mínimos cuadrados no ponderados, previo análisis de la prueba de Bartlett y el coeficiente Kaiser-Meyer-Olkin (KMO). El análisis paralelo sugirió un modelo unidimensional; para estos análisis se utilizó el programa FACTOR Analysis versión 10,1. En tercer lugar se ejecutó un Análisis Factorial Confirmatorio (AFC), mediante el software estadístico AMOS versión 21, con el cual se evaluó la bondad de ajuste del modelo y se utilizó el modelamiento de ecuaciones estructurales (SEM). La bondad de ajuste absoluta e incremental se determinó a través del índice de ajuste comparativo (CFI) y el índice de Tucker-Lewis Index (TLI). Asimismo, se utilizó los parámetros para el error cuadrático medio de aproximación (RMSEA) y el índice de error de cuadrático medio (RMR); todos estos bajo los criterios sugeridos por Hu y Bentler (Hu \& Bentler, 1999). Por último, se calculó la confiabilidad a través del coeficiente alfa de Cronbach y sus respectivos intervalos de confianza (Dominguez-Lara, 2016).

El presente estudio ha seguido las recomendaciones de la declaración de Helsinki en el desarrollo de sus objetivos. La participación de este estudio fue completamente voluntaria y se solicitó el consentimiento verbal de los participantes ante de enrolarse o formar parte del estudio. Posteriormente, se aseguró que la información contenida en el estudio sería totalmente anónima y que ellos no podrían ser reconocidos de ninguna manera. La información recolectada en este estudio solo se utilizó para los fines de esta investigación en particular.

\section{Resultados}

La Tabla 1 muestra los resultados de la evaluación de diez expertos, quienes analizaron la relevancia, representatividad y claridad de los ítems de la escala EFT-Cov19. Se aprecia que los ítems recibieron una evaluación favorable ( $\mathrm{V}>0,70)$ y en su mayoría, los ítems son relevantes, son importantes y claros (V = 1,00; IC 95\%: 0,89-1,00). Asimismo, se aprecia que todos los valores del límite inferior (Li) del IC 95\% son adecuados y todos los valores del coeficiente $\mathrm{V}$ fueron estadísticamente significativos. Por lo tanto, la escala EFT-Cov19 reporta evidencia de la validez basada en el contenido.

Tabla 1. V de Aiken para la evaluación de la relevancia, representatividad y claridad de los ítems de la escala EFT-Cov19

\begin{tabular}{|c|c|c|c|c|c|c|c|c|c|c|c|c|}
\hline \multirow{2}{*}{ Ítems } & \multicolumn{4}{|c|}{ Relevancia $(n=7)$} & \multicolumn{4}{|c|}{ Representatividad $(n=7)$} & \multicolumn{4}{|c|}{ Claridad $(n=7)$} \\
\hline & $\mathbf{M}^{*}$ & $\mathbf{D E}^{\dagger}$ & $\mathbf{V}^{\ddagger}$ & IC $95 \%$ § & $\mathbf{M} *$ & $\mathbf{D E}^{\dagger}$ & $\mathbf{V}^{\ddagger}$ & IC $95 \%$ \& & $\mathbf{M}^{*}$ & $\mathbf{D E}^{\dagger}$ & $\mathbf{V}^{\ddagger}$ & IC 95\% ${ }^{\S}$ \\
\hline Ítem 1 & 3,00 & 0,00 & 1,00 & $0,85-1,00$ & 3,00 & 0,00 & 1,00 & $0,85-1,00$ & 3,00 & 0,00 & 1,00 & $0,85-1,00$ \\
\hline Ítem 2 & 2,71 & 0,49 & 0,90 & $0,71-0,97$ & 2,71 & 0,49 & 0,90 & $0,71-0,97$ & 2,71 & 0,49 & 0,90 & $0,71-0,97$ \\
\hline Ítem 3 & 2,86 & 0,38 & 0,95 & $0,77-0,99$ & 3,00 & 0,00 & 1,00 & $0,85-1,00$ & 2,86 & 0,38 & 0,95 & $0,77-0,99$ \\
\hline Ítem 4 & 3,00 & 0,00 & 1,00 & $0,85-1,00$ & 3,00 & 0,00 & 1,00 & $0,85-1,00$ & 3,00 & 0,00 & 1,00 & $0,85-1,00$ \\
\hline Ítem 5 & 3,00 & 0,00 & 1,00 & $0,85-1,00$ & 3,00 & 0,00 & 1,00 & $0,85-1,00$ & 2,86 & 0,38 & 0,95 & $0,77-0,99$ \\
\hline Ítem 6 & 2,86 & 0,38 & 0,95 & $0,77-0,99$ & 3,00 & 0,00 & 1,00 & $0,85-1,00$ & 2,86 & 0,38 & 0,95 & $0,77-0,99$ \\
\hline Ítem 7 & 3,00 & 0,00 & 1,00 & $0,85-1,00$ & 3,00 & 0,00 & 1,00 & $0,85-1,00$ & 2,86 & 0,38 & 0,95 & $0,77-0,99$ \\
\hline Ítem 8 & 2,86 & 0,38 & 0,95 & $0,77-0,99$ & 2,86 & 0,38 & 0,95 & $0,77-0,99$ & 2,86 & 0,38 & 0,95 & $0,77-0,99$ \\
\hline Ítem 9 & 3,00 & 0,00 & 1,00 & $0,85-1,00$ & 3,00 & 0,00 & 1,00 & $0,85-1,00$ & 3,00 & 0,00 & 1,00 & $0,85-1,00$ \\
\hline
\end{tabular}

\section{Análisis preliminar de los ítems}

La Tabla 2, muestra la media, desviación estándar, asimetría y curtosis de los nueve ítems de la EFT-Cov19. Se observa que el ítem 4 tiene el mayor puntaje promedio $(M=3,39)$ y el ítem $6(\mathrm{DE}=1,30)$ muestra la mayor dispersión. Los valores de asimetría y curtosis no exceden el rango +/- 1,5 (Pérez \& Medrano, 2010). Y la correlación entre los ítems fueron significativas $(>0,30)$.

\section{Análisis Factorial Exploratorio (AFE)}

Se realizó un AFE previo análisis del índice Kaiser-Meyer-Olkin (KMO =0,903) y la prueba de esfericidad de Bartlett (1751.9; gl = 36; p < 0,001), los cuales fueron adecuados. Se utilizó el método de mínimos cuadrados no ponderados con rotación oblicua Promin, además, para la determinación de factores se utilizó el análisis paralelo, el cual reveló la existencia de un solo factor. La solución rotada de los nueve ítems explica el 58,17\% de la varianza total explicada. El Factor obtenido explica 44,3\% de la varianza y todos los ítems presentan saturaciones mayores a 0,60. 
Tabla 2. Análisis preliminar de los ítems de la EFT-Cov19

\begin{tabular}{|c|c|c|c|c|c|c|c|c|c|c|c|c|c|}
\hline \multirow[b]{2}{*}{ Ítems } & \multirow[b]{2}{*}{ Media } & \multirow[b]{2}{*}{$\begin{array}{l}\text { Desviación } \\
\text { Estándar }\end{array}$} & \multirow[b]{2}{*}{ Asimetría } & \multirow[b]{2}{*}{ Curtosis } & \multicolumn{9}{|c|}{ Correlación inter-ítem } \\
\hline & & & & & $\begin{array}{c}\text { Ítem } \\
1\end{array}$ & $\begin{array}{c}\text { Ítem } \\
2\end{array}$ & $\begin{array}{c}\text { Ítem } \\
\mathbf{3}\end{array}$ & $\begin{array}{c}\text { Ítem } \\
4\end{array}$ & $\begin{array}{c}\text { Ítem } \\
5\end{array}$ & $\begin{array}{c}\text { Ítem } \\
6\end{array}$ & $\begin{array}{c}\text { Ítem } \\
7\end{array}$ & $\begin{array}{c}\text { Ítem } \\
8\end{array}$ & $\begin{array}{c}\text { Ítem } \\
9\end{array}$ \\
\hline Ítem 1 & 2,147 & 1,099 & 0,564 & $-0,566$ & 1,000 & & & & & & & & \\
\hline Ítem 3 & 3,344 & 1,129 & $-0,253$ & $-0,809$ & 0,314 & 0,427 & 1,000 & & & & & & \\
\hline Ítem 4 & 3,395 & 1,137 & $-0,292$ & $-0,853$ & 0,328 & 0,436 & 0,716 & 1,000 & & & & & \\
\hline Ítem 5 & 2,359 & 1,181 & 0,575 & $-0,507$ & 0,423 & 0,396 & 0,452 & 0,427 & 1,000 & & & & \\
\hline Ítem 8 & 2,836 & 1,263 & 0,124 & $-1,046$ & 0,425 & 0,394 & 0,53 & 0,522 & 0,548 & 0,634 & 0,739 & 1,000 & \\
\hline Ítem 9 & 3,117 & 1,204 & $-0,089$ & $-0,97$ & 0,366 & 0,402 & 0,516 & 0,541 & 0,501 & 0,526 & 0,667 & 0,695 & 1,000 \\
\hline
\end{tabular}

Tabla 3. Análisis factorial exploratorio de la EFT-Cov19

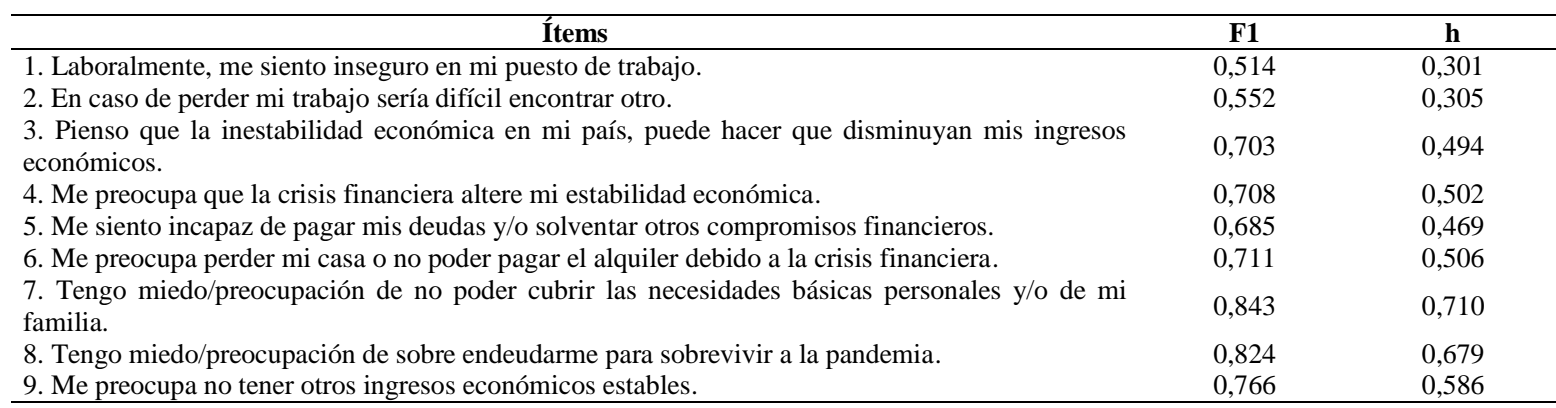

$$
\mathrm{F} 1=\text { Factor } 1 . \mathrm{h}=\text { comunalidades. }
$$

\section{Análisis factorial confirmatorio (AFC)}

Para verificar la evidencia de validez; basada en la estructura interna de la EFT-Cov19 se desarrolló un AFC, los resultados del primer modelo no presentaron índices de ajuste adecuados. Por lo que, a través de la técnica de modificación de índices, se eliminó los ítems 2 y 4, obteniendo índices adecuados para el modelo de un factor $\left(\chi^{2}=\right.$ 63,914, $\mathrm{df}=15, \mathrm{p}<0,001 ; \mathrm{IFI}=0,981 ; \mathrm{TLI}=0,971 ; \mathrm{CFI}=0,980 ; \mathrm{RMSEA}=0,069$ y $\mathrm{RMR}<0,05)(\mathrm{Tabla} 4)$. Las cargas factoriales estandarizadas para los siete ítems se pueden apreciar en la Figura 1.

Tabla 4. Índices de ajuste de los modelos evaluados por CFA del instrumento del estudio

\begin{tabular}{cccccccc}
\hline Modelo & $\boldsymbol{\chi}^{\mathbf{2}}$ & df & IFI & TLI & CFI & RMSEA & RMR \\
\hline 9 ítems & 365,452 & 27 & 0,902 & 0,869 & 0,902 & 0,129 & 0,075 \\
$\mathbf{7}$ ítems & 63,914 & 14 & 0,981 & 0,971 & 0,980 & 0,069 & 0,038 \\
\hline
\end{tabular}

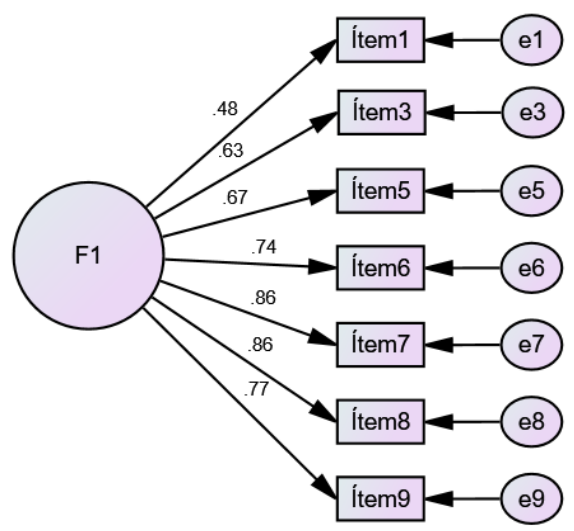

Figura 1. Modelo unifactorial de la Escala EFT-Cov19 
Validez convergente y discriminante

La validez convergente y discriminante se analizó mediante la correlación de Pearson entre los 3 test: EFTCov19, LABOR-P y WHO-5. La EFT-Cov19 correlacionó positivamente con la LABOR-PE $(\mathrm{r}=0,564, p<0,01)$ y con un tamaño del efecto mediano. Asimismo, la EFT-Cov19 se correlaciona de manera inversa y estadísticamente significativa con la WHO-5 $(r=-0,336, p<0,01)$ obteniendo un tamaño del efecto pequeño. Los hallazgos muestran evidencias de validez convergente y discriminante (Tabla 4).

Tabla 4. Medias, desviaciones estándar y correlaciones entre las escalas del WLEIS, GAD-2 y WHO-5

\begin{tabular}{lllll}
\hline Variable & M & DS & $\mathbf{1}$ & $\mathbf{2}$ \\
\hline 1. Escala EFT-Cov19 & 25,27 & 7,86 & & \\
2. LABOR-PE & 11,33 & 0,606 & $0,606^{* *}$ & \\
3. WHO-5 & 13,16 & $-0,336$ & $-0,336^{* *}$ & $-0,285^{* *}$ \\
\hline
\end{tabular}

Nota. $\mathrm{M}=$ media; $\mathrm{DS}=$ desviación estándar, ** indica $\mathrm{p}<0,01$

\section{Confiabilidad}

La fiabilidad de la EFT-Cov19 se calculó con el coeficiente $\alpha$ de Cronbach, obteniendo un valor aceptable $(\alpha=$ $0,896 ;$ IC $95 \%=0,88-0,90)$.

\section{Discusión}

La presente escala de 7 ítems encontró una adecuada distribución en un solo factor. Lo que permite por su brevedad poder ser aplicada rápidamente a la población objetivo a ser estudiada. Entre sus ítems, evalúa el estrés percibido por los trabajadores dependientes sobre su estabilidad laboral y económica durante la pandemia del COVID19. En ese sentido, los dos primeros ítems evaluaron la inseguridad y el estrés generado por la posibilidad de perder o tener inestabilidad en el puesto de trabajo donde actualmente laboran. Un estudio realizado por Lozano-Chagua et al observó que durante la pandemia del COVID-19 el estrés y la depresión aumentaron de manera vertiginosa; esto como consecuencia de la pérdida de empleos y el aislamiento social obligatorio (Chaguay et al., 2020). De manera que, la evaluación de estos parámetros cobra una importancia especial, sobre todo en el contexto de una emergencia sanitaria.

El presente estudio evaluó la validez convergente y discriminante de la escala EFT-CoV19, donde se mostró que al correlacionarla con la escala LABOR-PE se obtuvieron valores $r=0,564$. La búsqueda bibliográfica mostró que la gran mayoría de escalas que evalúan el estrés secundario a problemas económicos o responsabilidades laborales no presentan estudios de validez convergente y discriminante (Acuña et al., 2017; Lobo Dinis Castro et al., 2013). Sin embargo, Martínez Correa y Reyes del Paso realizaron un estudio con el fin de determinar la correlación convergente de una escala de riesgo en enfermedad somática posterior a procesos psicopatológicos causadas por noxas de diferentes tipos, en su estudio encontraron que la escala bajo los modelos de Eysenck y Grossarth-Maticek mostraron valores $\mathrm{r}$ de 0,17; 0,22; y 0,28, en los ítems 1, 3 y 6, respectivamente (Reyes del Paso \& Martínez Correa, 2004). A pesar de que, los instrumentos son difíciles de comparar por la diferencia en los objetivos perseguidos por cada uno de ellos, la comparación simple nos muestra que los elementos de la escala EFT-CoV19 muestra una correlación positiva superior a la mostrada por otros instrumentos psicométricos.

La escala EFT-CoV19 mostró una confiabilidad aceptable al tener valores de a de Cronbach de 0,896, lo que demuestra que es un instrumento confiable que responde a un solo factor. La literatura muestra pocas escalas que puedan ser comparables con el estudio presentado en este proyecto. Sin embargo, el estudio realizado por SalvadorMoreno guarda sintonía con los objetivos de la presente investigación. El equipo liderado por Salvador-Moreno diseñó una escala de Inserción de Salario Emocional, a diferencia de nuestra escala, el instrumento diseñado convergió en 3 factores que obtuvieron valores de a de Cronbach de 0,97; 0,96 y 0,98; para los 3 factores respectivamente (SalvadorMoreno et al., 2021). Los valores del alfa de Cronbach globales para el instrumento en conjunto fueron de 0,97, lo que le otorga una excelente confiabilidad. Es probable, que la buena confiabilidad responda a un mejor diseño de los instrumentos y una selección optima de la muestra necesaria para la validación de este instrumento. Aun así, los valores presentados en la escala EFT-CoV19 demuestran que es una escala confiable y reproducible en contextos similares al utilizado para su validación.

Las principales limitaciones del estudio se encontraron en el tipo de muestreo, que permitió la aparición de un sesgo de selección. Este sesgo impide que los hallazgos puedan ser extrapolables a poblaciones más grandes o en otros contextos diferentes al evaluado en este estudio (como, por ejemplo, en trabajadores de zonas rurales). Sin embargo, su aplicación y su revalidación en contextos mucho más grandes puede confirmar los hallazgos encontrados 
en este estudio. En ese sentido, su aplicación es recomendable en poblaciones que enfrentan situaciones de estrés a causa de las vicisitudes financieras que han acaecido como consecuencia de la pandemia del COVID-19.

En conclusión, la escala EFT-CoV19 es una escala valida y confiable para poder medir el estrés financiero en los trabajadores dependientes, esto como efecto de los eventos ocurridos por la pandemia del COVID-19.

\section{Conflicto de intereses}

Los autores declaran no tener conflicto de interés.

\section{Agradecimientos}

A los estudiantes que apoyaron en la recolección de las encuestas.

\section{Referencias}

Acuña, H. I., Michelini, Y. N., Guzman, J. I., \& Godoy, J. C. (2017). Evaluación de la validez convergente y discriminante en pruebas informatizadas de toma de decisiones. Avaliacao Psicológica, 16(3):375-383. https://doi.org/10.15689/ap.2017.1603.12952

Alola, A. A., Alola, U. V., \& Sarkodie, S. A. (2020). The nCOVID-19 and financial stress in the USA: Health is wealth. Enviroment, Development and Sustainability, 12. https://doi.org/10.1007/s10668-020-01029-w

Ato, M., López-García, J. J., \& Benavente, A. (2013). Un sistema de clasificación de los diseños de investigación en psicología. Anales de Psicología, 29(3):1038-1059. https://doi.org/10.6018/analesps.29.3.178511

Campo-Arias, A., Pedrozo-Cortés, M. J., \& Pedrozo-Pupo, J. C. (2020). Escala de estrés percibido relacionado con la pandemia de COVID-19: Una exploración del desempeño psicométrico en línea. Revista Colombiana de Psiquiatría, 49(4): 229-230. https://doi.org/10.1016/j.rcp.2020.05.005

Ceballos-Vásquez, P., Cancino-Grillo, M., González-Palacios, Y., \& Paravic Klijn, T. (2020). Perfil de riesgos psicosociales en trabajadores sanitarios: Una herramienta elemental para su intervención. Revista de la Asociación Española de Especialistas en Medicina del Trabajo, 29(4):323-329. Disponible en: https://scielo.isciii.es/scielo.php?script=sci_arttext\&pid=S1132-62552020000400323 (Acceso marzo 2021)

Chaguay, L. A. L., Chaguay, S. L., \& Galeas, R. R. (2020). Desempleo en tiempos de COVID-19: Efectos socioeconómicos en el entorno familiar. Journal of Science and Research: Revista Ciencia e Investigación. 5(4): 187-197. Disponible en: https://revistas.utb.edu.ec/index.php/sr/article/view/926 (Acceso febrero 2021)

Delgado-Fernández, V., Rey-Merchán, M. del C., Arquillos, A. L., Delgado-Fernández, V., Rey-Merchán, M. del C., \& Arquillos, A. L. (2021). Estudio comparativo de los riesgos psicosociales laborales entre profesionales médicos. Revista de la Asociación Española de Especialistas en Medicina del Trabajo, 30(1):24-33. Disponible en: https://scielo.isciii.es/scielo.php?script=sci_arttext\&pid=S1132-62552021000100024 (Acceso enero 2021)

Doern, R. (2021). Knocked down but not out and fighting to go the distance: Small business responses to an unfolding crisis in the initial impact period. Journal of Business Venturing Insights, 15:e00221. https://doi.org/10.1016/j.jbvi.2020.e00221

Dominguez-Lara, S. A. (2016). Datos normativos de la Escala de Procrastinación Académica en estudiantes de psicología de Lima. Revista Evaluar, 16(1):20-30. https://doi.org/10.35670/1667-4545.v16.n1.15715

Ganga Contreras, F., \& Villacís Moyano, H. (2018). Factores individuales y grupales que influyen en la productividad de los trabajadores de las grandes y medianas empresas de Guayaquil. Revista Perspectivas, 42:97-122. Disponible en: http://www.scielo.org.bo/scielo.php?pid=S1994$37332018000200005 \&$ script $=$ sci_abstract\&tlng=pt (Acceso enero 2021)

Hernández González, A. I., Ortega Andeane, R. P., \& Reidl Martínez, L. M. (2012). Validación del instrumento de estrés laboral para médicos mexicanos. En-claves del pensamiento, 6(11):113-129.m Disponible en: http://www.scielo.org.mx/scielo.php?script=sci_arttext\&pid=S1870-879X2012000100007 (Acceso enero 2021)

Hertz-Palmor, N., Moore, T. M., Gothelf, D., DiDomenico, G. E., Dekel, I., Greenberg, D. M., Brown, L. A., Matalon, N., Visoki, E., White, L. K., Himes, M. M., Schwartz-Lifshitz, M., Gross, R., Gur, R. C., Gur, R. E., Pessach, I. M., \& Barzilay, R. (2021). Association among income loss, financial strain and depressive symptoms during COVID-19: Evidence from two longitudinal studies. Journal of Affective Disorders, 291:1-8. https://doi.org/10.1016/j.jad.2021.04.054 
Hu, L., \& Bentler, P. M. (1999). Cutoff criteria for fit indexes in covariance structure analysis: Conventional criteria versus new alternatives. Structural Equation Modeling: A Multidisciplinary Journal, 6(1):1-55. https://doi.org/10.1080/10705519909540118

Lobo Dinis Castro, M. M., Marques Hökerberg, Y. H., \& Lambert Passos, S. R. (2013). Validade dimensional do instrumento de qualidade de vida WHOQOL-BREF aplicado a trabalhadores de saúde. Cadernos de Saúde Pública, 29(7):1357-1369. https://doi.org/10.1590/S0102-311X2013000700010

Mamani-Benito, O., Apaza Tarqui, E. E., Carranza Esteban, R. F., Rodríguez-Alarcón, J. F., \& Mejía, C. R. (2020). Inseguridad laboral en el empleo percibida ante el impacto del COVID-19: Validación de un instrumento en trabajadores peruanos (LABOR-PE-COVID-19). Revista de la Asociación Española de Especialistas en Medicina del Trabajo, 29(3):184-193. Disponible en: https://scielo.isciii.es/scielo.php?script=sci_arttext\&pid=S1132-62552020000300002 (Acceso enero 2021)

Mejia, C. R., Reyes-Tejada, A. L., Gonzales-Huaman, K. S., Leon-Nina, E. C., Murga-Cabrera, A. X., CarrascoAltamirano, J. A., Quispe-Cencia, E., Mejia, C. R., Reyes-Tejada, A. L., Gonzales-Huaman, K. S., Leon-Nina, E. C., Murga-Cabrera, A. X., Carrasco-Altamirano, J. A., \& Quispe-Cencia, E. (2020). Riesgo de estrés post traumático según ocupación y otros factores durante la pandemia por COVID-19 en el Perú. Revista de la Asociación Española de Especialistas en Medicina del Trabajo, 29(4):265-273.

Mejía Córdova, G. (2017a). Pérdida de productividad en el lugar de trabajo relacionada con el estrés financiero. Journal of Behavior, Health \& Social Issues, 8(2):25-34. http://dx.doi.org/10.1016/j.jbhsi.2017.06.001

Mejía Córdova, G. (2017b). Relación entre Estrés Financiero y el Bienestar de los Empleados de Petróleos Mexicanos en el sureste de México. Investigación administrativa, 46(119), 1-10.

Organización Internacional del Trabajo. (2020a). El teletrabajo durante la pandemia de COVID-19 y después de ella. OIT. Disponible en: https://www.ilo.org/wcmsp5/groups/public/---ed_protect/---protrav/--travail/documents/publication/wcms 758007.pdf (Acceso enero 2021)

Organización Internacional del Trabajo. (2020b). Gestión de los riesgos psicosociales relacionados con el trabajo durante la pandemia de COVID-19. OIT. Disponible en: https://www.ilo.org/wcmsp5/groups/public/--ed_protect/---protrav/---safework/documents/instructionalmaterial/wcms 763314.pdf (Acceso enero 2021)

Pérez, E. R., \& Medrano, L. A. (2010). Análisis factorial exploratorio: Bases conceptuales y metodológicas. Revista Argentina de Ciencias del Comportamiento ( RACC ), 2(1), 58-66.

Reyes del Paso, G. A., \& Martínez Correa, A. (2004). Personalidad y factores clásicos de riesgo en la predicción de la enfermedad somática: Un análisis de la validez convergente y discriminante del modelo de Eysenck y GrossarthMaticek en sujetos análogos. Análisis y modificación de conducta, 30(131):349-403. Disponible en: http://hdl.handle.net/11162/21717 (Acceso enero 2021)

Robillard, R., Saad, M., Edwards, J., Solomonova, E., Pennestri, M.-H., Daros, A., Veissière, S. P. L., Quilty, L., Dion, K., Nixon, A., Phillips, J., Bhatla, R., Spilg, E., Godbout, R., Yazji, B., Rushton, C., Gifford, W. A., Gautam, M., Boafo, A., ... Kendzerska, T. (2020). Social, financial and psychological stress during an emerging pandemic: Observations from a population survey in the acute phase of COVID-19. BMJ Open, 10(12): e043805. https://doi.org/10.1136/bmjopen-2020-043805

Salvador-Moreno, J. E., Torrens-Pérez, M. E., Vega-Falcón, V., \& Noroña-Salcedo, D. R. (2021). Diseño y validación de instrumento para la inserción del salario emocional ante la COVID-19. RETOS. Revista de Ciencias de la Administración y Economía, 11(21):41-52. https://doi.org/10.17163/ret.n21.2021.03

Topp, C. W., Østergaard, S. D., Søndergaard, S., \& Bech, P. (2015). The WHO-5 Well-Being Index: A Systematic Review of the Literature. Psychotherapy and Psychosomatics, 84(3):167-176. https://doi.org/10.1159/000376585

Vásquez-Trespalacios, E. M., Aranda-Beltrán, C., \& López-Palomar, M. del R. (2020). Síndrome de Burnout y justicia organizacional en profesionales de enfermería de hospitales de tercer nivel en la ciudad de Medellín. Revista de la Asociación Española de Especialistas en Medicina del Trabajo, 29(4):330-339. Disponible en: https://scielo.isciii.es/scielo.php?script=sci arttext\&pid=S1132-62552020000400330 (Acceso febrero 2021) 